Para enlazar con este artículo / To link to this article:

http://dx.doi.org/10.14198/fem.2017.29.06

Para citar este artículo / To cite this article:

García-Arroyo, Ana. «Realidades y paradojas en la Educación Superior de India: (in)visibilidad de la mujer». En Marcos Jesús Iglesias Martínez e Inés Lozano Cabezas (coords.), La (in)visibilidad de las mujeres en la Educación Superior: retos y desafios en la Academia. Feminismo/s, 29 (junio 2017): 153-171, DOI: 10.14198/fem.2017.29.06

\title{
REALIDADES Y PARADOJAS \\ EN LA EDUCACIÓN SUPERIOR DE INDIA: (IN)VISIBILIDAD DE LA MUJER
}

\author{
REALITIES AND PARADOXES OF INDIA'S HIGHER \\ EDUCATION: WOMAN'S (IN)VISIBILITY
}

\author{
Ana GARCÍA-ARROYO \\ Universitat Rovira i Virgili \\ garciaarroyo7@gmail.com \\ orcid.org/0000-0001-6720-6319
}

\section{Resumen}

En este artículo me propongo analizar los principales problemas de la Educación Superior india, centrándome en los cambios que se han producido con la globalización, a principios de los años noventa, y cómo afectan directamente a las mujeres. Analizaré los datos más relevantes que se desprenden del informe del Gobierno indio del año 2014-15, All India Survey on Higher Education 2014-15, qué representan dichas cifras y a qué realidades y paradojas corresponden, en las que se ve implicada la mujer. Basándome en la filosofía sobre Educación Superior de Martha Nussbaum (2010), el objetivo final de mi investigación es demostrar que las consecuencias derivadas de la implantación de un sistema de Educación Superior, basado en el modelo occidental utilitario y dirigido hacia el mercado, han afectado a la mujer india y continuarán amenazando su calidad académica y su equidad de género-casta-clase.

Palabras clave: mujer india, globalización, educación utilitarista, consecuencias presentes y futuras.

\begin{abstract}
This article analyzes the most important problems of India's Higher Education. It focuses on the changes that have been produced from the arrival of globalization at the beginning of the 1990s until now, which have direct implications on women. I will deal with the most relevant data in All India Survey on Higher Education 2014-15 by
\end{abstract}


the Ministry of Higher Education (Indian Government), paying special attention to what these figures stand for in terms of representing the realities and paradoxes that affect women. Based on Martha Nussbaum's philosophy on Higher Education (2010), the ultimate aim of this research is to demonstrate that the consequences derived from the implementation of a system of Higher Education, based on the market-oriented utilitarian Western model, have directly affected Indian women and will continue threatening their academic quality and their gender-class-caste equality.

Keywords: Indian woman, globalization, utilitarian education, present \& future consequences. 


\section{INTRODUCCIÓN Y OBJETIVOS}

Desde la independencia de India en 1947 y con la implementación de la Constitución, la educación se ha considerado un derecho por excelencia para la población india, independientemente de su casta, religión, género o sexo. Por esta razón, para las castas tradicionalmente más vulnerables (Scheduled Castes) y los grupos tribales también desprotegidos (Scheduled Tribes), se estableció una cuota de admisión en educación y en puestos de trabajo, en las instituciones del Estado. Durante todos estos años el gobierno indio se ha esforzado considerablemente en reforzar el sistema educativo a través de sus diversos planes quinquenales, centrándose principalmente en la educación obligatoria, aunque la Educación Superior también ha dado un salto considerable si tenemos en cuenta que en 2011 había 42 universidades centrales, 275 universidades estatales, 130 universidades deemed ${ }^{1}$ y 90 universidades privadas, a las que además se ha de añadir un número elevado de colleges, públicos y privados. En los últimos años el énfasis principal ha recaído en la potenciación de las áreas de ciencia y tecnología, así como en educación a distancia, que ha dado como resultado el hecho de que los Institutos Superiores de Tecnología, de Comercio, de Empresariales, junto con la Jawaharlal Nehru University en Delhi, hayan obtenido un reconocimiento académico a nivel mundial por su prestigiosa educación. Sin duda, con la mejora de la economía y, sobretodo, desde que India abre sus puertas a los mercados internacionales a principios de los 1990s, con la introducción de las tecnologías de la comunicación y de internet, la educación, en todos sus niveles, pero principalmente la Educación Superior, ha sufrido un cambio radical. También es en este momento cuando el sector privado interviene, creando sus propias universidades, escuelas e institutos, que llegan a abarcar hoy en día el $60 \%$ del total de las instituciones educativas (Shagur). Por otro lado, a pesar de que unas determinadas instituciones académicas indias destaquen a nivel mundial, la otra realidad es que la calidad del sistema de Educación Superior indio se ha cuestionado desde diferentes

1. Universidad deemed (Deemed University) es un término utilizado en India para aludir a una institución de educación superior del mismo nivel de enseñanza que la Universidad.

Feminismo/s 29, junio 2017, pp. 153-171 
posturas y sigue siendo objeto de un arduo debate. Consecuentemente, en el plan diseñado por el gobierno que comprende desde el 2012 hasta el 2017 se ha propuesto como objetivo principal mejorar la calidad de la Educación Superior en todo el país. Si tenemos en cuenta la gran complejidad ${ }^{2}$ de un país como India, podríamos incluso afirmar que, a pesar de que todavía quede mucho por hacer, ha habido una evolución muy positiva con respecto a la Educación Superior y se ha logrado en muy poco tiempo. Por otro lado, sabemos que India está constantemente cambiando y mejorando en derechos sociales y civiles, en todos los campos, aunque, desafortunadamente, todavía sigue siendo un país eminentemente patriarcal. Por esta razón, desde la mirada de los Estudios de Género, una se pregunta qué papel ha tenido la mujer ${ }^{3}$ en el mundo educativo de la Enseñanza Superior, bien como estudiante o como profesora, si ha evolucionado su posición dentro de la institución académica, cuáles son sus principales logros y los problemas mayores a los que se ha enfrentado y si en las expectativas de futuro que el gobierno contempla para la educación y la persecución del 'progreso' también se incluye a la mujer, es decir, si en las mejoras de la calidad académica (quality), también se incluyen mejoras de igualdad de género (equality).

En este artículo me propongo analizar los principales problemas de la Educación Superior india, centrándome en los cambios que se han producido con la globalización, a principios de los años noventa, y cómo afectan a las mujeres. Analizaré los datos más relevantes que se desprenden del informe del Gobierno indio en el año 2014-15, qué representan dichas cifras y a qué realidades y paradojas corresponden, en las que se ve implicada la mujer. El objetivo final de mi

2. Utilizo el término «complejidad» para definir India y referirme a los 1.200 millones de habitantes, sus miles de castas, múltiples cultos religiosos, diferentes etnias, miles de lenguas (oficiales y no oficiales), multiplicidad de tradiciones y costumbres, es decir, toda su diversidad multicultural que hacen del país un lugar muy complejo para gobernar.

3. Este artículo huye de todo tipo de esencialismos y generalizaciones y quiere aclarar que utiliza la terminología de «la mujer india» o «las mujeres indias» indistintamente. Se es consciente y se subraya la complejidad, la diversidad y la diferencia con respecto a 'la mujer' y/o 'las mujeres' indias. Al mismo tiempo la autora considera que 'la mujer india' / 'las mujeres indias', así como 'la mujer', en general, en el mundo de hoy, en cualquier país o cultura, en su diversa condición de raza, clase, casta etc., sigue estando sometida a las normas hegemónicas patriarcales y neocolonialistas locales, nacionales e internacionales, en un grado que puede variar muy ampliamente, según cada persona en particular, y del que ella misma, la mujer, puede tener consciencia de ello o no tenerla plenamente. Esto no significa -como subraya Spivak- que la mujer, como sujeto subalterno, esté desempoderada y no pueda hablar, contestar y desafiar el discurso colonizador; al contrario, la realidad ha demostrado que es lo que ha venido haciendo desde épocas ancestrales y continua haciendo hoy, alterando ese discurso hegemónico y manifestando sus deficiencias y absurdidades. 
investigación es demostrar que las consecuencias derivadas de la implantación de un sistema de Educación Superior, el cual está basado en el modelo occidental utilitario y va dirigido hacia el mercado, ha afectado a la mujer y continuará amenazando su calidad académica y su equidad de género-casta-clase.

\section{LA «INDIA RESPLANDECIENTE» DE LA GLOBALIZACIÓN: PROBLEMAS DE LA EDUCACIÓN SUPERIOR}

Como la gran mayoría de países del mundo, India está inmersa en la atmósfera desenfrenada y mareante de conseguir 'el progreso' a través de un modelo económico competitivo y global. Su actuación hasta ahora ha sido ejemplar y se plasma en su imparable crecimiento económico. El McKinsely Quarterly apunta que si continúa manteniendo el ritmo de crecimiento, para el año 2025 se convertirá en la quinta economía consumidora del mundo (Beinhocker et al.). La revista The Economist también mantiene que India tiene un crecimiento económico mucho más elevado que ningún otro país y seguirá así durante las dos próximas décadas ( 2010 a \& 2010 b). Sin embargo, esta imagen victoriosa de una «India Resplandeciente» (Shining India) también revela otra cara más oscura si nos detenemos a examinar las vidas de millones de personas que no se benefician del actual modelo de crecimiento económico vertiginoso. Todo lo contrario, este modelo económico que ha dado lugar al dominio hegemónico de los Estados Unidos y que ha sido imitado por todo el mundo occidental, ofreciendo sus repercusiones a nivel global, en todos los demás países, poco o casi nada se ha preocupado por establecer una política de igualdad y calidad de derechos, por ejemplo, los relacionados con el género, la raza y la casta principalmente. La filósofa americana Martha Nussbaum, a la que se le concedió el Premio Príncipe de Asturias en 2012 por su labor en el campo de las Ciencias Sociales y las Humanidades, una gran seguidora de la obra del Premio Nobel de Literatura indio, Rabindranath Tagore, demuestra todo esto en su estudio Not for Profit:

Today's India offers a revealing laboratory of such experiments, as some states (Gujarat, Andhra Pradesh) have pursued economic growth through foreign investment, doing little for health, education, and the condition of the rural poor $[\ldots]$ Achievements in health and education, for example, are very poorly correlated with economic growth [...] So producing economic growth does not mean producing democracy. Nor does it mean producing a healthy, engaged, educated population in which opportunities for a good life are available to all social classes (15).

Un siglo antes de que Nussbaum escribiese estas palabras, Rabindranath Tagore, el gran maestro no sólo en el arte literario sino también en la construcción de 
un modelo alternativo de Universidad, donde prima la creatividad y no la competencia y la economía, cuestionaba ya el mercantilismo desbocado y el afán de 'progreso', como serias amenazas para la humanidad y la educación (Tagore).

En la «India Resplandeciente», que quiere estar a la altura en el mercado global, hay también un sentimiento de inquietud debido a las infraestructuras tan pobres con las que todavía cuenta el país, por ejemplo, en lo que respecta a la Educación Superior. En Deepening Democracy Madhu Kishwar comenta que si dejamos a un lado algunos Institutos y Escuelas de Educación Superior, como he mencionado arriba, los/as estudiantes indios/as no acaban sus carreras universitarias con una buena preparación, capaces de ocupar puestos relevantes y sacar a India de la corrupción y la ineficiencia. Kishwar también subraya que los sucesivos gobiernos indios han descuidado y en ocasiones destruido una serie de oficios y conocimientos ancestrales, en lugar de haber modernizado todas esas técnicas y haber proveído a estos profesionales con una Educación Moderna competente. Kishwar subraya que al adoptar un sistema de educación superior basado en el modelo académico inglés, que se introdujo en el país en el siglo XIX, en el que el inglés se convierte no sólo en la lengua dominante, sino en un signo de educación elitista, vinculada a las profesiones más distinguidas, todas aquellas personas que no tienen acceso a una excelente educación en inglés quedan relegadas a un segundo término y son tratadas, en muchos casos, de ignorantes y analfabetas. Consecuentemente, hoy en día, muchos de los estudiantes que van a la Universidad, especialmente los varones, se inclinan hacia carreras que les proporcionen mucho dinero, o que les brinden la posibilidad de optar a puestos administrativos del estado que otorgan prestigio social. Y, sin embargo, estos estudiantes no poseen el talento y conocimiento suficientes para competir en el mercado global, señala Kishwar.

Según el profesor Ram Takwale, los problemas principales de la Educación Superior india de hoy se pueden resumir en los siguientes puntos: a) el mercantilismo de la educación, es decir, el bien social de la educación se ha convertido en objeto de mercado y tiene esta orientación exclusiva, con lo cual se desprestigia toda aquella formación que no va dirigida hacia el mercado y no sea rentable para el gobierno; b) la competitividad global, que significa elevar la calidad y el estándar para poder entrar en una carrera imparable de agresiva competición a nivel mundial; c) débil conexión entre el mundo educativo y el trabajo; d) grandes disparidades de calidad y estándares mínimos en las diferentes instituciones académicas; y e) costes muy caros que ofrecen como resultado la imposibilidad de acceso para un gran número de personas de las clases medias y bajas. 
Fazal Rizvi y Radhika Gorur también comparten estos argumentos con respecto a la Educación Superior india: «a system that remains in a poor shape, characterized by inadequate infrastructure, poor operating conditions and ineffective teaching and learning programs, producing large cohorts of graduates who are barely employable in the professions for which they are ostensibly been trained»(2). En el 2007, incluso el mismo ministro de Desarrollo de Recursos Humanos, Arjun Singh, comparó el sistema de Educación Superior con un «niño enfermizo» en el periódico The Hindu (2007), que no ha mejorado demasiado a pesar de las reformas introducidas en los últimos años. Es muy relevante subrayar que estas reformas no han tenido el éxito que originalmente se esperaba, no porque estuviesen mal encaminadas, o por la falta de recursos económicos, sino más bien porque han chocado contra un conjunto de paradojas y realidades socio-culturales que se resisten a los grandes cambios y que están relacionadas con la casta, la etnia, la clase social, la región y el género. Un ejemplo muy claro lo encontramos en las disparidades e inequidades concernientes a la mujer india que paso ahora a analizar.

\section{REALIDADES Y PARADOJAS: ¿POR QUÉ SE PRODUCEN?}

Primeramente, quiero subrayar una vez más que cuando nos referimos al concepto de 'mujer india', no se debe concebir como un conjunto o categoría homogénea, pues caeríamos en los reduccionismos hegemónicos típicos del colonialismo, que bien apuntó Mohanty en su famoso artículo «Under Western Eyes», entre otros/as estudiosos/as (2008). La clase social, la casta, el grupo tribal y la región son algunos de los factores más importantes que contribuyen a la diferencia y heterogeneidad de las mujeres indias, que también están presentes cuando nos referimos a la Educación Superior. Por otro lado, también es bien cierto que, en términos generales, la mujer india, como sujeto social y político, no disfruta de un estado superior o igual al del hombre y estas mismas categorías de clase, casta, religión, etc., sólo contribuyen a multiplicar sus desventajas e inequidades en todos los terrenos, incluido el de la Educación Superior. Ya he apuntado anteriormente que en la Educación Superior india existe una línea divisoria situada al inicio de los años noventa, que marca el antes y el después de la globalización, que ha transformado el modelo educacional y ha derivado en tremendas consecuencias de género. Karuna Chanana en «Subject Choices and Gender: Women in Higher Education in India» (2005) arguye que desde los 1950s hasta los noventa, el objetivo del gobierno se centraba en identificar los problemas principales de la educación de la mujer, tratar de solventarlos y buscar vías de conexión entre la Universidad y la vida laboral posterior. Igualmente, Sahni and Shakar en «Girls' Higher Education 
in India on the Road to Inclusiveness» arguyen que durante los primeros años después de la independencia india las aulas estaban abarrotadas de estudiantes varones, con lo que la labor del gobierno fue promover la participación de la mujer en la Educación Superior. Incluso las políticas de educación de 1986, conocidas como The National Policy of Education, se propusieron, férreamente, empoderar a las mujeres a través de la educación con el fin de solucionar disparidades e inequidades de género ${ }^{4}$. Un resultado muy positivo lo tenemos en el incremento del número de mujeres matriculadas en la Universidad: entre 1950-55 la cifra era tan sólo del 10,9\% mientras que en el curso de 1991-92 alcanzó el 32\% (Chanana 2005). La mujeres entraban en carreras consideradas tradicionalmente femeninas como Magisterio y Humanidades, incluyendo esta última Lenguas, Literaturas y Ciencias Sociales, dejando a un lado los otros estudios 'más masculinos' como Comercio, Derecho e Ingeniería. En las carreras de Ciencias, un 28.8 \% de mujeres se llegó a matricular en 1980-81 y en Medicina las mujeres alcanzaron una cifra del $31,7 \%$ en $1988-89$. En este último caso, Medicina, debo añadir que en India nunca ha sido una carrera tradicionalmente masculina debido al hecho de que al existir la costumbre, en algunas comunidades, de segregar a las mujeres y alejarlas del contexto de los hombres, también esta misma restricción cultural ha exigido mujeres-médico y ha favorecido el acceso de la mujer india a estos estudios universitarios.

Con el cambio impactante que se produce a partir de 1991, las políticas del gobierno se caracterizan por disminuir la inversión en Educación Superior, realizar una re-estructuración de las universidades públicas y fomentar la autofinanciación de las instituciones académicas a través de capitales privados. También se permite la creación de instituciones de Educación Superior totalmente privadas y autónomas, sin unas normas por parte del gobierno indio que regulen su funcionamiento, para que de esta manera puedan hacer frente al aumento de la demanda de cursos y carreras específicas (Anandkrishnan). La globalización en India, como en el resto del mundo, trae además un aumento de las carreras científico-técnicas orientadas hacia el mercado, en detrimento de las disciplinas que tradicional y mayoritariamente han sido la opción de las mujeres como las Humanidades y las Ciencias Sociales. La división entre Letras y Ciencias se acentúa aún más, pues en la India global las Ciencias se subdividen en Ciencias Puras y Ciencias Aplicables, creándose así una jerarquía

4. Recordemos aquí que ya antes de la independencia, durante la etapa colonial en el siglo XIX, los reformadores indios pusieron sus objetivos en las reformas y mejoras que afectaban a la mujer, liberándolas de tradiciones ancestrales opresoras y dotándolas de educación. Ver: Historia de las mujeres de la India (2009) de Ana García-Arroyo. 
de disciplinas más precisa que dificulta más el acceso y la equidad de la mujer en estas instituciones superiores.

Por otro lado, la globalización y todos sus cambios sociales han conseguido que algunas mujeres indias, por su contexto familiar y social, también se hayan lanzado a realizar estudios de las nuevas disciplinas académicas como apunta Chanana en «Gender and Disciplinary Choices» (2004):

The influence of the so-called economic liberalization and the market demand on women's access to higher education as well as women's choices of subjects because one sees a perceptible change in the choices of women at least in the metropolitan cities in India where they are flocking to the new 'profesional' courses such as management, fashion designing, computers, human resource management etc. (8.)

Según el estudio de Chanana, en el curso 1993-94 el 54,2\% de las mujeres se matriculaba en las carreras de letras, sólo el 19,8\% lo hacía en las de Ciencias y un porcentaje todavía más bajo, el 14,6\% se decantaba por Comercio (Commerce).

Si echamos ahora una ojeada al último informe del Gobierno indio, All India Survey on Higher Education (2014-15), facilitado por el Department of Higher Education, obtenemos una serie de datos más actuales que confirman lo que se está demostrando en este artículo. En el curso 2014-15, el total de los estudiantes varones matriculados en la Educación Superior india era del 54\% frente al 45,5\% de mujeres que lo hacían. Estas cifras representan un 25,3\% de hombres y un 23,2\% de mujeres que son estudiantes entre 18-23 años que acceden a estudios superiores en todo el país.

Me gustaría detenerme ahora en las carreras principales en que estos/as estudiantes se matriculan y el nivel, ya que para este mismo año se observa que el porcentaje más elevado de estudiantes aparece en primer lugar en el Grado de Letras (Bachelor of Arts), seguido del Grado de Comercio (Bachelor of Commerce) y finalmente del Grado de Ciencias (Bachelor of Science). Las cifras también demuestran que la liberalización económica ha facilitado a las mujeres el acceso a las nuevas carreras o a aquellos terrenos considerados tradicionalmente masculinos como Empresariales, Ingeniería, Diseñadora de moda, Informática, Comercio, Recursos Humanos, Tecnologías de la Información, etc., especialmente si éstas habitan en zonas urbanas. No obstante, el número sigue manteniéndose bastante inferior, comparado con el de los estudiantes varones. Algunos ejemplos lo confirman: en el Grado de Ciencias hay un 52,4\% de hombres y sólo un 37,2\% de mujeres; en el Grado de Comercio las cifras son de 54,5\% de hombres y 45,5\% de mujeres; en el Grado de Tecnología el índice se dispara con $73 \%$ de hombres y tan sólo $27 \%$ de mujeres; y en el Grado de 
Ingeniería existe un $71,5 \%$ de varones y un $24,5 \%$ de mujeres. Este Grado de Ingeniería comprende disciplinas como Electrónica, Informática, Ingeniería Mecánica, Ingeniería Electrónica y similares.

Sin embargo, las carreras en las que las mujeres siguen ofreciendo un número de matrículas más elevado son el Grado en Magisterio con un 64\% frente al 36\% de varones, y el Grado de Letras con un 53\% de mujeres y un 47\% de estudiantes varones. También en la carrera de Medicina siguen destacando las mujeres con un 55\% por la razón cultural que he comentado antes. En este Grado de Medicina se incluyen además otras ramas como Farmacia, Medicina Ayurvédica y Odontología.

Es interesante también comprobar que según este informe del gobierno indio de 2014-15, si nos fijamos en el acceso a la Educación Superior por estados indios, se demuestra la tendencia también tradicional, en este caso favorable a las mujeres, de un número más elevado de matrícula para ellas en el estado de Kerala, frente al $42 \%$ de hombres matriculados. En realidad es el único estado indio en el que hay mayor acceso de la mujer al estamento educativo superior, es decir, se confirma el hecho de que Kerala ha sido un estado en el que ha existido una conciencia de mayor igualdad y equidad de derechos de género, que se ha manifestado desde tiempos ancestrales con la existencia del sistema matriarcal y matrilineal mantenido hasta la época moderna; después, durante la India independiente, siempre ha existido esa particular lucha del pueblo por eliminar las costumbres opresoras y hacer que mejoren y prevalezcan los derechos de la mujer

El mismo informe también hace relación al porcentaje total de estudiantes que pertenecen a las distintas castas de intocables (Scheduled Castes) y a las poblaciones tribales (Scheduled Tribes) que logran acceder a los estudios superiores, con sólo un $13,5 \%$ y un $4,8 \%$ respectivamente. No hace especificaciones por género, pero una puede imaginar que el número de mujeres que logran acceder a la Educación Superior es muy bajo.

Teniendo estas cifras presentes la pregunta que una se hace ahora es, ¿por qué ocurre todo esto? ¿Por qué con la globalización y la expansión educativa se ha producido un incremento de las mujeres en la Educación Superior pero éstas optan, en su mayoría, por carreras consideradas tradicionalmente femeninas? ¿Cuál es el contexto indio y las paradojas del mismo sistema que condicionan estas decisiones? A continuación me propongo dar respuestas a estos interrogantes y otros derivados de ellos.

5. Ver, por ejemplo, la obra literaria de la escritora india Kamala Das, donde se narra el poder y, al tiempo, la naturalidad del sistema matriarcal. También ver, «Legacies of Matriliny: The Place of Women and the Kerala Model» de Robin Jeffrey. 
1) Existen una serie de factores físicos que ponen difícil el acceso de la mujer a la Educación Superior como pueden ser la falta de instituciones académicas, la carencia de infraestructuras adecuadas para la mujer, sobre todo en las zonas más rurales y provinciales, como alojamiento económico (hostels), lavabos femeninos y espacios para ellas; carencia de modelos diferentes de mujer que transciendan el patrón tradicional; número bajo de profesoras y de becas; falta de un mayor número de materias en el currículum académico para su elección; carencia de profesorado femenino en puestos de poder (rectorado, administración) con políticas de equidad; reglas excesivamente restrictivas para la mujer. Mi propia investigación en 2008 y 2010 en algunos Colleges de West Bengal y en la Universidad de Rohtak, en el estado de Haryana, confirma lo que expongo ${ }^{6}$. En algunas zonas las infraestructuras son inadecuadas en todos los niveles académicos y humanos. No se contemplan ni las mínimas necesidades básicas de la mujer, como la existencia de lavabos para ellas donde se pueda tener un poco de intimidad y seguridad. Esto demuestra que aún se conciben los estudios universitarios como un espacio masculino, innecesario para la mujer que debe ceñirse a su rol tradicional de esposa y madre. Por otro lado también pude constatar como evidencia que la educación en la Jawaharlal Nehru University en Nueva Delhi es excelente en términos de calidad, como he mencionado arriba, pero si consideramos la equidad, principalmente de género, se siguen produciendo grandes abusos por la carencia de dichas políticas.

Los estudios anteriores de Sahni y Kale por un lado, y de Samal por otro, ya confirmaban mi investigación. Estos autores demuestran que la Educación Superior india no es igual en todas partes del país y establecen diferencias entre zonas: las urbanas/semi-urbanas y las rurales. Como ejemplo citan el estado de Orissa donde en el momento de su estudio había 50 colleges del gobierno en las zonas urbanas, pero ninguno en las rurales.

Otra disparidad a nivel estatal y que vincula directamente al género la encontramos entre los estados del norte de India y los del sur. En el sur el aumento de matrícula de estudiantes mujeres ha coincidido con el incremento de las instituciones académicas por parte del Gobierno, y de capitales

6. La autora se ha dedicado a los Estudios de India durante más de veinte años, con numerosas estancias y viajes por el país, dando como resultado diversos ensayos, traducciones y obra creativa, algunos de los cuales se han apuntado aquí. Ha realizado investigación y ha sido profesora invitada y conferenciante en diversas ocasiones, en distintos lugares de India, por ejemplo, Trivenidevi Bhalotia College, Barjora College, Michael Madhusudan Memorial College, Visva-Bharati University en West Bengal; también en la Universidad de Rohtak y en la Jawaharlal Nehru University; así como en la Pune University. Todo ello le ha permitido establecer un constante diálogo con estudiantes y profesorado de excelencia que ha enriquecido su investigación. 
privados, que han fomentado carreras especializadas y orientadas al mercado. Sin embargo, en los estados del norte las matrículas han subido, hay más mujeres en la Universidad, pero los centros académicos no han aumentado (Sahni y Shakar). De esto se deriva que: «if a girl aspires for the profesional streams, she would have to (1) compete for limited locally available seats or (2) migrate to institution abundant zones in 'south'. While the first option has competitive hurdles, the second option is equally hindered by the resistance to female migration» (244). En otras palabras, que el lugar de donde una mujer india procede o habita también la ofrece mayores o menores desventajas con respecto a las posibilidades de su Educación Superior.

En algunas zonas del norte de India como Rajasthan, Bihar, Jharkhand, Uttar Pradesh, donde la pobreza es más punzante y las prácticas socio-culturales discriminan a la mujer en todos los terrenos, es difícil lograr una actitud positiva de las familias con respecto a la educación de sus hijas. Por un lado está la costumbre cultural de excluir a la mujer de la esfera pública y limitarla al ámbito privado, que se práctica más en los estados del norte que en los del sur. Por otro lado, estos estados del norte han tenido una influencia mayor de los textos ancestrales hegemónicos y brahmánicos, como la épica del Mahabharata y del Ramayana, y el tratado legal de Las Leyes de Manu, que han definido las normas patriarcales, los roles tradicionales para el hombre y la mujer, y la familia patriarcal, que siguen teniendo un peso primordial en nuestros días (García-Arroyo 2009). Se requiere, por tanto, que para mejorar la equidad de género en el futuro tiene que haber una mayor visibilidad y participación de las mujeres en las instituciones superiores educativas y en los puestos académicos y administrativos de poder, en estos estados que tradicionalmente ofrecieron mayor resistencia a los cambios y mejoras en general y, concretamente, las centradas en las propias mujeres.

2) Frecuentemente lo que ocurre es que aquellos que gobiernan las distintas instituciones de Educación Superior tienen una mente muy patriarcal, excesivamente estereotipada, con barreras de género fijas, estáticas y tradicionales, sobre todo en las zonas rurales y provinciales, que no ayuda para que la mujer india tenga un fácil acceso a la educación académica y la integración en el contexto. Un ejemplo sería el hecho de que las estudiantes mujeres participen en juegos y deportes típicos del campus universitario, como el cricket o el fútbol, algo muy común entre los estudiantes varones y que el sistema académico desaprueba por la influencia socio-cultural.

La mente patriarcal también se refleja en la constante discriminación y violencia de género que se produce en las instituciones superiores. El abuso sexual y el acoso, a todos los niveles, siguen siendo muy comunes. Muy a menudo, 
los cargos con poder como vice-rectores, profesores y otros directivos, acosan a sus estudiantes y al profesorado femenino. La prensa india frecuentemente publica este tipo de injusticias porque las mismas mujeres acosadas se resisten al silencio de tiempos pasados y son ellas mismas las que denuncian estas agresiones abiertamente como leemos en The Chronicle of Higher Education, «On India's Campuses, Female Students Speak Out About 'Deep Rooted' Gender Discrimination» (Najar).

3) Otro exponente de esta cultura patriarcal es que en las familias tradicionales, los padres tienden a priorizar el matrimonio frente a la Educación Superior de su hija. Lo que suele ocurrir es que si la familia es de clase social media o alta, es decir, con unos determinados recursos económicos, hoy en día, automáticamente, se asume que irá a la Universidad y recibirá estudios superiores. Orientada por la familia, la joven se matriculará en un Grado de Letras y/o Humanidades y el diploma conseguido al finalizar los estudios servirá como recurso para elevar su estatus social a la hora de buscar marido. Estas familias tradicionales de clase media o alta también conciben la educación de la hija como un respaldo en el caso de que el matrimonio falle, por ejemplo, si la mujer es abandonada por el marido, si fuese necesario el divorcio o si se quedase viuda.

En las familias de recursos más limitados ni siquiera se plantea la dicotomía de elegir entre ahorrar para la educación de la hija o para su dote. Obviamente, la educación supone una inversión considerable de dinero y saben que las carreras de Letras no garantizan que ese dinero invertido dé algún fruto en el futuro y retorne a través de un puesto de trabajo. En las carreras más especializadas, como los Grados en Tecnología, en Ingeniería y todos los que tienen que ver con las Ciencias Aplicadas, la educación supone una inversión muy elevada que estas familias de pocos recursos económicos no pueden costear. Además, se ha de tener también en cuenta que aunque la hija cursase una carrera -cualquiera que fuese- y posteriormente obtuviese un trabajo que compensase todos los gastos invertidos en su educación, según la tradición cultural estos ingresos no irían nunca a parar a la familia de la hija, sino a la del novio cuando se casase.

También se ha de mencionar que ya en la educación Primaria, Secundaria y Bachillerato, muchas de estas familias tradicionales aleccionan a la hija para que no se decante por asignaturas consideradas 'masculinas'. Tampoco se gastarán el dinero en proporcionarles buenas escuelas o en darles clases extraordinarias y lecciones privadas para que ésta obtenga una excelente educación que la haga destacar en las pruebas de nivel y acceso a las instituciones superiores (Chanana 2004). De nuevo, deducimos que la mente patriarcal, 
todavía muy anclada en las viejas costumbres culturales, está bien presente en la vida contemporánea india del siglo XXI.

4) La interrelación de todos estos factores socio-culturales, espaciales y de género que intervienen en la Educación Superior repercute directamente en el mercado laboral donde observamos que, una vez más, las mujeres son menos visibles que los hombres y donde las mismas paradojas e inequidades aparecen abrumadoras. Tomemos como ejemplo el estudio de Louise Morley y Barbara Crossouard, Women in Higher Education Leadership in South Asia, basado en el año académico 2011. Estas estudiosas demuestran que en este año el porcentaje de mujeres académicas en la Universidad india era del 35,7\% frente al 64,3\% de hombres. Si observamos la distribución de mujeres por estados las cifras más elevadas corresponden a Chandigarh (55\%), Meghalaya (53\%), Kerala (52,3\%) y Goa $(49,5 \%)^{7}$. Las cifras más bajas se sitúan en Bihar (15,3\%), Arunachal Pradesh (16,6\%), Jharkhand (26,3\%) y West Bengal $(27,1 \%)$, todos ellos situados en la mitad norte de India. Si nos detenemos a examinar qué puestos académicos ocupa el profesorado según las diferentes escalas se constata, una vez más, que en todas las categorías los porcentajes de las mujeres-profesoras son inferiores a los de sus colegas varones. En la posición más alta de catedrático/a y/o titular existe un 74,5\% de varones frente a la ridícula cifra de 25,5\% de académicas. En los puestos intermedios de Lectorado y/o Profesorado Asociado tenemos un $68,9 \%$ de hombres y un $31,1 \%$ de mujeres. En la plaza más baja de Ayudante (Lecturer / Assistant) hay un 61,5\% de hombres y un $38,5 \%$ de mujeres ${ }^{8}$.

Si comparamos estas cifras con los datos que apunta el informe del Gobierno indio en All India Survey on Higher Education para el año 2014-15 con un $61 \%$ de hombres-profesores en la Universidad y el 39\% de mujeres académicas, deducimos que en tres años de diferencia, del 2011 al 2014, la cifra ha subido un poco a favor de las mujeres, pero sin ni siquiera llegar a la mitad. También es alarmante el número de académicos/as correspondientes a castas bajas de intocables (Scheduled Caste) el 7.1\%, de tribales (Scheduled Tribes) el $2.1 \%$ y de las 'Otras castas pobres' (OBC) el 23\% que han logrado

7. Chandigarh no es un estado, sino una ciudad muy moderna perteneciente al estado de Haryana, pero aparece en el estudio por poseer la cifra más alta. He de comentar también que está situada al norte, igual que el estado de Meghalaya y, por tanto, sería una paradoja más, frente a la realidad expuesta de que los estados del norte suelen ser más tradicionales, donde las inequidades de género son más latentes.

8. Ver Devi, la intocable de Ana García-Arroyo, una novela ambientada en hechos reales que recorre la vida de una mujer intocable y los problemas con los que se enfrenta para acceder a la educación, así como los abusos que tiene que soportar cuando finalmente logra ser profesora (2017 -en proceso de edición-). 
un puesto en la Educación Superior, aunque no se especifique el porcentaje de hombres y de mujeres.

Aunque sabemos que los datos estadísticos pueden variar ligeramente, según las lecturas que se hagan de ellos, sí que reflejan una evidencia abrumadora y preocupante. Por un lado las políticas de Educación Superior del Gobierno indio y los distintos campus académicos deben poner en marcha, y con urgencia, programas de mayor sensibilización de género, visibilidad y empoderamiento de la mujer, estudiante o profesora, de forma que transcienda a la sociedad en general. Por otro lado, el sistema educativo en todas sus etapas debe poner todo su empeño en eliminar las barreras sociales y culturales que impiden a las castas y grupos sociales más desprotegidos (Scheduled Castes, Scheduled Tribes, $O B C$ ) acceder a la educación. Se deben establecer las medidas necesarias para que los comités de selección no estén formados todos por hombres, como suele ocurrir en la mayoría de casos, para que haya trasparencia y exista una paridad y unas normas de selección inclusivas que no discriminen a la mujer, cuando ésta posee requisitos iguales o mejores que sus homólogos varones. Si estos procesos de selección y/o promoción son honestos, transparentes y equitativos, no cabe duda de que la mujer -sea estudiante o académica- tendrá un fácil acceso a cargos y espacios de liderazgo donde pueda intervenir más eficazmente y lograr que las férreas estructuras patriarcales se tambaleen y caigan.

\section{CONCLUSIONES}

Llegados a este punto me gustaría concluir este artículo subrayando que las realidades y paradojas presentadas aquí correspondientes a la (in)visibilidad de la mujer en la Educación Superior en India manifiestan un complejo y multidimensional entramado de barreras sociales, culturales, económicas y estructurales, propias de un país donde el patriarcado todavía se resiste a transmutar y transferir su poder a favor de otras formas, maneras y estructuras alternativas que miran hacia la equidad y la contemplación de los Derechos Humanos. No cabe duda de que la liberalización económica y el propósito desenfrenado del Gobierno indio de ponerse a la altura mundial y conseguir el 'progreso' a través de un modelo económico competitivo no ha logrado que las grandes desigualdades que ya existían desapareciesen o disminuyesen. Es cierto que se ha incrementado el número de mujeres en la Universidad, pero la visibilidad de estas, el empoderamiento y la equidad todavía dejan mucho que desear. Indudablemente, existe la tendencia cada vez más elevada de que las hijas se formen adecuadamente y su educación les brinde una independencia y un puesto de trabajo. De hecho, entre las miles de caras que India nos muestra 
hoy, una concretamente es que cada día se ven más mujeres ocupando puestos en la vida pública, sobre todo en las ciudades, por ejemplo, en bancos, agencias de viajes, universidades, compañías multinacionales, agencias de información, hoteles, hospitales, etc.,, para los cuales es necesario una educación profesional específica y cara. Esto demuestra que desde los años noventa ha habido una transformación social y cultural en la sociedad, en general, y en las familias, en particular, que se ve palpable al menos en un sector de la sociedad. Las expectativas de los padres con respecto a sus hijas y las aspiraciones y sueños de éstas han cambiado. Aunque esta tendencia sea minoritaria y, podría decirse, clasista, ya que está amparada por una estructura mental abierta y un estatus económico aceptable, capaz de hacer frente a una educación relativamente cara, como se ha demostrado en este artículo, el cambio se ha iniciado y una parte de las mujeres indias se han subido al carro del mundo global y de la liberalización del mercado, gracias a la educación especializada recibida. No obstante, no debemos olvidarnos, como se ha mostrado aquí con el ejemplo del profesorado académico, que estas mujeres ocupan puestos inferiores a los de los hombres y aparecen representadas en cifras escasas, siendo casi invisibles en los cargos de poder. Tampoco debemos olvidarnos de las otras tres cuartas partes de la sociedad que hasta ahora no han podido optar a esta Educación Superior 'elitista' / clasista, orientada hacia el mercado y, teóricamente, hacia el 'éxito' y 'prestigio'. ¿Con qué planes cuenta el Gobierno para hacer frente a las innumerables paradojas e inequidades de género y sus intersecciones con la casta, raza, región, etc., en el futuro? Según la Federation of Indian Chambers of Commerce and Industry y su informe Higher Education in India: Vision 2030, en esta fecha se aspira a que India se haya convertido en «a globally dominant economy, with a high quality higher education sector that leads and fulfills the needs of society» (3) $)^{9}$. Mi investigación cuestiona estas expectativas de la Cámara de Comercio e Industria india pues, basándome en lo que se ha demostrado aquí, me decanto más por apoyar y subscribir el pensamiento principal de Martha Nussbaum, de donde he partido, marcado por la idea de que perseguir el crecimiento económico, 'el progreso', como señal de mejora del país, no implica mejorar la democracia, ni las oportunidades que deben tener todas las personas -y no sólo una minoría- con respecto a la educación,

9. Siguiendo la trayectoria anterior, este informe de la Federación de las Cámaras de Comercio e Industria (Federation of Indian Chambers of Commerce and Industry) plantea las metas a conseguir en la Educación Superior para el 2030 basándose en el modelo europeo caracterizado por 1) educación centrada en el alumno/a; 2) énfasis en la tecnología; 3) sistema competitivo y de acreditaciones; 4) internalización de la Universidad; 5) universidad de excelencia. 
el trabajo y los Derechos Humanos en general. ¿De qué sirve la consecución del máximo rendimiento económico si no existe una distribución equitativa de esa riqueza y la relación entre las personas sigue presentando grandes inequidades de género-casta-clase, como se ha reflejado aquí. ¿Quién querría vivir, en el 2030, en un país muy próspero donde no hay libertades ni derechos equitativos para todos sus habitantes? Nussbaum demuestra que el mundo contemporáneo se ha dejado llevar por ese ansia de competición feroz para conseguir el 'progreso' que le ha llevado a una profunda crisis de valores éticos y humanos que son esenciales para la democracia de cualquier país, incluido India como he mostrado aquí.

Desde los años noventa el Gobierno indio ha potenciado una Educación Superior utilitarista, orientada hacia el mercado. La cuestión, como he expuesto arriba, es que parece ser que continuará en la misma línea, incluso potenciándola, según se desprende de la visión de futuro que la Federation of Indian Chambers of Commerce and Industry planea conseguir para el año 2030. Mantener una Educación que se centra principalmente en la producción del mayor beneficio económico conlleva una gran amenaza para la democracia en general, y para la equidad de género en particular, como este artículo ha demostrado. El Gobierno indio está perdiendo la batalla al situarse a favor de la violenta competencia global con fines mercantilistas y su consecuente deshumanización, en lugar de potenciar la igualdad y el respeto a través de un modelo educativo alternativo como, por ejemplo, el que ya inició y practicó en el siglo pasado Rabindrananth Tagore con exitosos resultados. Un modelo que incite a vivir la vida con pensamientos y sentimientos de respeto, igualdad y empatía hacia las demás personas, pues ningún sistema de educación puede ser saludable si sólo beneficia a las minorías poderosas, mientras excluye, invisibiliza y arrincona al resto de la población, especialmente a las mujeres.

\section{REFERENCIAS BIBLIOGRÁFICAS}

All India Survey on Higher Education 2014-2015. New Delhi: Goverment of India. Ministry of Human Resources and Development. Department of Education, 2016. <http://aishe.nic.in/aishe/viewDocument.action?documentId=206>, consultado el 2-10-2016

Anandkrishnan, M. «Private Investments in Technical Education». Private Iniciatives in Higher Education. New Delhi: Sneh Prakashan and Amity Foundation for Learning, 2004.

Beinhocker, Eric, Diana Farrell y Adil Zainulbhai. «Tracking the Growth of India's Middle Class». McKinsey Quaterly. August, 2007. 
Chanana, Karuna. «Gender and Disciplinary Choices: Women in Higher Education in India». The UNESCO Colloquium on Research and Higher Education Policy. Paris, 2004.

Chanana, Karuna. «Subjects Choices and Gender: Women in Higher Education in India». Writing the Women's Movement. A Reader. Ed. Mala Khullar. Delhi: Zubaan, 2005, 269-289.

Das, Kamala. My Story. New Delhi: Harper Collins, 2009.

García-Arroyo, Ana. Historia de las mujeres de la India. Barcelona: Laertes, 2009.

García-Arroyo, Ana. Devi, la intocable. Madrid: Kailas, 2017. En proceso de edición. «Higher Education, a Sick Child». The Hindu. 11 Octubre, 2007.

Higher Education in India: Vision 2030. Federation of Indian Chambers of Commerce and Industry. Summit 2013. <http://www.ey.com/Publication/vwLUAssets/ Higher-education-in-India-Vision-2030/\%24FILE/EY-Higher-education-inIndia-Vision-2030.pdf>, consultado el 20-10-2016

Jeffrey, Robin. «Legacies of Matriliny: The Place of Women and the Kerala Model». Pacific Affairs. University of British Columbia. 77.4 (2005): 647-664.

Kishwar, Madhu. Deepening Democracy. Challenges of Governance and Globalization in India. Oxford University Press, 2005.

Mohanty, Chandra. «Under Western Eyes. Feminist Scholarship and Colonial Discourses». The Post-colonial Studies Reader. Eds. Bill Ashcroft, Gareth Griffiths \& Helen Tiffin. London: Routledge, 2008, 259-263.

Morley, Louise y Barbara Crossouard. Women in Higher Education Leadership in South Asia: Rejection, Refusal, Reluctance, Revisioning. University of Sussex. Centre of Higher Education and Equality Research. <https://www.britishcouncil.org/sites/default/files/morley_crossouard_final_report_22_dec2014.pdf >, consultado el 1-11-2016

Najar, Nida. «On India's Campuses, Female Students Speak Out about 'Deep Rooted' Gender Discrimination». The Chronicle of Higher Education. 22 Abril, 2013. <http://www.chronicle.com/article/Female-Scholars-Describe-Deep/138659/ consultado el 15-11-2016

Nussbaum, Martha. Not for Profit: Why Democracy Needs the Humanities. Princeton University Press, 2010.

Rizvi, Fazal y Radica Gorur. «Challenges Facing Indian Higher Education». Australia India Institute. Two, Winter, 2011.

Sahni R., y S. Kale. «GATS and Higher Education: Some Reflections». Economic and Political Weekly 39 (2004).

Sahni, R., y V. K. Shakar. "Girls' Higher Education in India on the Road to Inclusiveness: On Track but Heading Where?» The International Journal of Higher Education Research 63 (2012): 237-256.

Samal, K. Equity and Financing Education. Globalization and Challenges for Education. NIEPA, 2003. 
Shagur, Obadya Ray. «Higher Education in India. Access, Equity, Quality». EAN World Congress, 2013.

Tagore, Rabindranath. Selected Essays. New Delhi: Rupa \& Co., 2010.

Takwale, Ram. «Challenges and Opportunities of Globalization for Higher Education in India. Alternatives through e-Education». New Delhi: University Grants Commision. Diciembre, 2003. <http://www.ugc.ac.in/oldpdf/pub/lectures/ugc_pro2.pdf >, consultado el 12-10-2016

«Business in India: A Bumpier but Freer Road». The Economist. 20 Sep 2010. <http://www.economist.com/node/17145035>, consultado el 2-11-2016

«India's Surprising Economic Miracle». The Economist. 30 sept 2010. < http://www.economist.com/node/17147648 >, consultado 2-11-2016 